\title{
Soluções criativas: o uso de Recursos Educacionais Abertos no acesso e na democratização do Ensino Superior
}

\author{
Ramón Garrote Jurado * \\ Marlene Zwierewicz ${ }^{* *}$ \\ Roberto Moraes Cruz ${ }^{* * *}$ \\ Tomas Pettersson ${ }^{* * *}$
}

\section{Resumo}

A ampliação da Educação a Distância - EaD no contexto brasileiro e internacional vem sendo acompanhada por uma série de desafios, implicando, minimamente, na necessidade de compatibilidade entre a oferta, as possibilidades pedagógicas para o uso de Recursos Educacionais Abertos - REA e as especificidades de aprendizagem de cada contexto. Considerando a relevância da EaD para o acesso e a democratização do Ensino Superior, este estudo tem como objetivo refletir sobre desafios enfrentados na criação ou adaptação de propostas de ensino para a modalidade ou projetos semipresenciais, destacando soluções que permearam esse processo em diferentes iniciativas impulsionadas pela Universidade de Borås - HB (Suécia). Trata-se, portanto, de um relato de experiências que apresenta soluções criativas planejadas a partir dos desafios observados na implantação de propostas em diferentes países, cuja intenção é colaborar para que outras instituições e outros profissionais interessados facilitem o processo de inserção da $\mathrm{EaD}$.

Palavras-chave: e-learning, Recursos Educacionais Abertos, Ensino Superior.

Creatuve Solutons: the use of Open Educational Resources in the access and democratization of Higher Education

\footnotetext{
Abstract

The increase of Distance Education (DE) in national and international context has been accompanied by a series of challenges involving the need for compatibility between supply, the pedagogical possibilities for the use of Open Educational Resources (OER), and the specifics of each learning context. Considering the relevance of DE to the access and democratization of Higher Education, this study aims to ponder over the challenges faced in the creation or adaptation of

* Doutor em Educação. Professor da Universidade de Borås - HB (Suécia). E-mail: ramon.garrote@hb.se

** Doutora em Educação e Doutora em Psicologia. Professora do Centro Universitário Barriga Verde UNIBAVE (Santa Catarina/Brasil). E-mail: marlenezwie@unibave.net

*** Doutor em Psicologia. Professor da Universidade Federal de Santa Catarina - UFSC (Brasil). E-mail: robertocruzdr@gmail.com

**** Mestre em Educação. Professor do Unicentro Borås (Suécia). E-mail: pettersson@telia.com
} 
teaching proposals for distance education or blended projects, highlighting solutions which permeated this process in different initiatives promoted by the University of Borås - HB (Sweden). It is, therefore, an account of experiences that presents creative planned solutions from the challenges observed in the implementation of proposals in different countries, intended to collaborate with other institutions and interested professionals to facilitate the insertion process of DE. Keywords: e-learning. Open Educational Resources. Higher education.

Introdução

No desenvolvimento atual das organizações, há um estímulo ao trabalho colaborativo. Isso se deve pelas possibilidades que ele oferece para a motivação, o apoio e o reconhecimento mútuos. Especificamente na área educacional, o trabalho em rede proporciona possibilidades inéditas, especialmente quando os professores se apoiam no uso das Recursos Educacionais Abertos - REA para dinamizar as práticas de ensino e os projetos de pesquisa e extensão.

É nesse processo de uso cada vez mais intenso de recursos tecnológicos para fins educativos (KARGIDIS et al., 2003) que mudar de paradigma educacional pode ser, ao mesmo tempo, desafiador e gratificante (SCOTT, 1999). Enquanto se observa a capacidade dos Recursos Educacionais Abertos - REA para o acesso ao ensino, colaborando para sua democratização, é indiscutível que a compreensão adequada ao utilizá-los contribui para que sejam explorados de acordo com as necessidades e potencialidades de cada contexto.

Xie e Ke (2011) afirmam que intercâmbios colaborativos sobre temas das unidades curriculares de um curso podem ter um impacto substancial na experiência da aprendizagem, enquanto Blin e Munro (2008), Britain e Liber (1999), Bush e Mott (2009), Findik e Ozkan (2010), Garrote Jurado (2007, 2012), Irwin e Berge (2006), Ladyshewsky e Gardner (2008), Ubell (2000) e Wilson e Stacey (2004) têm sugerido que as ferramentas de interação podem melhorar o rendimento escolar, em função das possibilidades que oferecem para a criação de comunidades de aprendizagem.

A utilização de Recursos Educacionais Abertos - REA, cada vez mais intensiva, também tem contribuído para que a Educação a Distância - EaD dissemine-se em vários setores, não somente no Ensino Superior, mas também na Educação Básica (MAIA; MATTAR, 2007). Este estudo, contudo, concentra-se em seus usos no Ensino Superior, delimitando seu foco, inicialmente, nos desafios enfrentados na adaptação de propostas de ensino para a $\mathrm{EaD}$ ou iniciativas semipresenciais.

Além dos desafios, o estudo também prioriza soluções, especialmente as viven- 
ciadas durante a trajetória profissional do primeiro autor deste artigo, Ramón Garrote Jurado. A seleção das experiências deve-se às transformações presenciadas durante seus anos de atuação no Ensino Superior, período em que o uso de Recursos Educacionais Abertos - REA vem se ampliando na área educacional, conforme destacado por Maia e Mattar (2007).

Diante de possibilidades e desafios, o estudo oferece aos docentes e demais profissionais do Ensino Superior, bem como às próprias instituições, reflexões sobre os problemas que podem emergir durante e depois da adaptação ou criação de propostas de $\mathrm{EaD}$ ou semipresenciais. A apresentação não se limita, entretanto, a uma simples descrição, mas pretende servir de alerta sobre os riscos de fracasso que ocorrem na implementação da EaD quando não são consideradas as compatibilidades entre a oferta, as possibilidades pedagógicas no uso de Recursos Educacionais Abertos - REA e as necessidades de aprendizagem de cada contexto.

\section{A relevância da EaD no acesso e na democratização do Ensino Superior}

Não é intenção deste estudo aprofundar a história da EaD, pois nosso objetivo está centrado na difusão de desafios e soluções para o acesso e a democratização do Ensino Superior. Consideramos, no entanto, oportuno delimitar alguns marcos que denotam a ampliação de seu uso.

Alves (2011) organizou uma síntese dos marcos históricos da $\mathrm{EaD}$ a partir das contribuições de estudos publicados por Vasconcelos, em 2010, e de estudos de Golvêa e Oliveira, publicados em 2006. Entre os principais marcos, a autora destaca o anúncio do primeiro curso com utilização de material para ensino e tutoria por correspondência, que ocorreu na Gazeta de Boston, em 1728; a inauguração do Instituto Liber Mermondes, na Suécia, em 1829, que possibilitou o acesso a cursos em EaD a mais de 150.000 pessoas; e o início da modalidade no contexto brasileiro, em 1904, com a publicação no Jornal do Brasil do anúncio da primeira oferta de profissionalização por correspondência para datilógrafo.

Além desses momentos, a autora registra outros marcos que complementam o quadro da ampliação da oferta dessa modalidade de ensino. Ainda que parte das iniciativas não tivesse como foco o Ensino Superior, elas foram fundamentais para criar condições de acesso e democratização da referida etapa formativa para pessoas em diferentes países.

Entre as possibilidades de ensino oferecidas atualmente, a $\mathrm{EaD}$ "[...] pode ser considerada a mais democrática das modalidades de educação, pois ao se utilizar de Tecno- 
logias de Informação e Comunicação transpõe obstáculos à conquista do conhecimento." (ALVES, 2011, p. 90). A autora também registra que a modalidade tem ampliado sua colaboração na democratização do ensino pela sua capacidade de atender, simultaneamente, um grande número de pessoas, por chegar a indivíduos que estão distantes dos centros de formação presencial e pela flexibilidade promovida nos horários de estudo.

Nascimento e Ferreira reforçam a relevância da EaD como uma "[...] possibilidade de política pública para a democratização do ensino e, em particular, o superior [...]" (2012, p. 2010). Ao contextualizar a constatação, os autores também indicam sua capacidade de oferecer o acesso a um maior número de pessoas, sem que se desloquem para centros de estudo ou enfrentem outros problemas como os decorrentes da condição financeira.

É nesse processo de avanço gradativo que os Recursos Educacionais Abertos - REA assumem um papel de relevância. Ao tornar possível uma modalidade de ensino que permite uma configuração diferenciada em relação à presencial, contribuem para que a EaD se transforme em uma "[...] alternativa viável à democratização das oportunidades educacionais no país, compreendendo a democratização como acesso, permanência e qualidade de ensino." (PRETI, 2005, p. 32).

Essa trajetória de ampliação foi facilitada pela criação de Sistemas de Gestão de Aprendizagem (SGA) e impulsionada pela criação dos referidos Recursos Educacionais Abertos (OER ou REA em português). Ao serem abordados a seguir, esses conceitos alavancam uma modalidade de educação que se torna cada vez mais acessível, em função da redução dos custos e de outros aspectos que democratizam o acesso e estimulam a permanência de estudantes no Ensino Superior, na Educação Básica e nos cursos extracurriculares.

Sistemas de Gestão de Aprendizagem - SGA e Recursos Educacionais Abertos REA

Os Sistemas de Gestão de Aprendizagem - SGA e os Recursos Educacionais Abertos - REA têm aproximações que permitem visualizar compatibilidades em seu uso. Disponibilizam também especificidades que são destacadas nas experiências apresentadas neste estudo, em razão das possibilidades que o último conceito oferece para o acesso e democratização do ensino, seja ele superior ou não.

Terminologicamente, existe uma diversificação nas expressões que nomeiam os SGA, tais como Sistema de Gestão da Aprendizagem ou Entornos de Ensino Virtual, com uso habitual das siglas em inglês "LMS" e "VLE", respectivamente. Um SGA é 
uma ferramenta TIC adequada para a comunicação de estudantes, docentes e outros participantes, sendo útil também para distribuir material das disciplinas e trabalhar documentos conjuntamente, além de possibilitar a realização de avaliações digitais, entre outras funções (BRITAIN; LIBER, 1999; DUTTON; CHEONG; PARK, 2004; SEEGER; ÅSTRÖM, 2005; GARROTE JURADO; PETTERSSON, 2007; GARROTE JURADO, 2012). Atualmente, existe uma grande oferta de programas com essa proposta, tanto os comercializáveis (WebCT, por exemplo) como os de código aberto (Moodle).

No mercado, observa-se uma disponibilização de produtos variados nessa linha, sendo que as especificidades são geradas por fusões entre companhias desenvolvedoras de aplicações para Internet, tais como a ocorrida entre BlackBoard e WebCT, dois dos SGA dominantes do mercado, nos quais o inglês é a língua de referência. Dado que o idioma utilizado em um SGA pode ser uma importante argumentação para sua aplicação, muitos países nos quais a língua inglesa não é o idioma materno desenvolvem seus próprios SGA.

Uma das especificidades que distingue os dois conceitos implicados neste estudo é a forma pela qual o recurso é ofertado. Enquanto um SGA pode ser comercializável ou aberto, o REA é de acesso gratuito. Portanto, "[...] um REA é simplesmente um recurso educacional com uma licença que facilita o seu reuso - e, possivelmente, adaptação - sem necessidade de solicitar a permissão do detentor dos direitos autorais." (BUTCHER, 2011, p. 34). Com a criação dos REA, a EaD conquista um importante recurso para facilitar o acesso ao ensino e, em decorrência, sua democratização.

\section{Experiências na Universidade de Borås - HB no ensino a distância e semipresencial}

As experiências apresentadas na sequência descrevem situações vivenciadas em diferentes contextos. A seleção dos exemplos tem como base o potencial que oferecem para mostrar que, se não forem consideradas as possibilidades pedagógicas do uso de ferramentas TIC e as necessidades de aprendizagem de cada contexto, existe um grande risco de fracasso na implementação da EaD.

Projeto Vinculação de Universidades da Ásia e Europa na Implementação de Tecnologia Sustentável, 2003 - 2006

A Universidade de Borås - HB coordenou o projeto Linkin Universities in Asia and Europe in Implementing Sustainable Technology (Vinculação de Universidades 
da Ásia e Europa na Implementação de Tecnologia Sustentável) entre os anos de 2003 - 2006. O projeto foi financiado pela Comunidade Europeia e articulado com o programa Asia Link.

Foram três as universidades que participaram do projeto: Universidade de Borås - HB (Suécia), Universidade de Reading (Grã-Bretanha) e Universidade Gadjah Mada (Indonésia). Essa experiência interinstitucional teve como finalidade o desenvolvimento conjunto das universidades participantes em um programa de Mestrado, com duração de dois anos e um total de 120 créditos.

As experiências desse projeto foram muito valiosas para as universidades participantes e também para as pessoas envolvidas: coordenadores, docentes e técnico-administrativos. Além de ampliar os contatos profissionais e pessoais entre os participantes, o projeto proporcionou um aumento da cooperação em trabalhos desenvolvidos nos anos posteriores.

Uma das pretensões do projeto era utilizar o ensino flexível e a distância dentro do programa de Mestrado oferecido pelo próprio projeto, para que tanto estudantes como professores das universidades dos três países pudessem estar interconectados durante seu desenvolvimento por meio de ferramentas TIC.

A iniciativa serviria para criar uma plataforma utilizável no incremento da compreensão e da comunicação em amplos espaços geográficos e culturais, dando suporte ao desenvolvimento sustentável do projeto. Por isso, entre as metas estavam: a utilização do ensino flexível, o desenvolvimento de aulas a distância em tempo real, a comunicação ativa entre docentes/estudantes e a avaliação da aprendizagem entre os próprios cursistas.

Para viabilizar o projeto, foi adquirida uma sala TIC para a Universidade de Gadjah Mada, com o propósito de que os docentes pudessem gravar aulas e enviá-las à Universidade de Borås - HB para sua distribuição. O equipamento foi adquirido para suprir limitações de banda larga dos serviços de Internet da Universidade de Gadjah Mada, o que dificultava as atividades em tempo real, como as teleconferências. A Universidade de Borås - HB também adquiriu um espaço de gravação que, infelizmente, nunca pôde ser configurado com o protocolo de Internet (TCP-IP) da universidade.

Observou-se, nessa experiência, que os problemas técnicos não foram suficientemente avaliados desde o princípio, fato que repercutiu em dificuldades como as que resultaram da conectividade das universidades colaboradoras do projeto, em função da incompatibilidade entre ferramentas TIC, tecnicamente muito avançadas, e capacidade de Internet. A utilização de tais ferramentas também exigia significativos recursos econômicos e humanos. 
Entre as soluções adotadas, decidiu-se por utilizar um SGA. Novas soluções, todavia, dão lugar a novos problemas. A utilização de um SGA no ensino exige que os docentes adaptem sua forma de docência, sendo que foi necessário incorporar ao projeto cursos e workshops voltados à formação dos docentes para o emprego do sistema.

Podemos concluir que o projeto começou a superar os obstáculos, originados pelo emprego das TIC no ensino, quando se optou pela formação docente e por um ensino a distância que entrelaçou as aulas (gravadas, telecomunicação e outras) e o uso de um SGA. Quando o projeto foi concluído, os professores puderam participar de outras ações, nas quais o conhecimento apropriado em relação ao emprego da técnica de um SGA no ensino foi decisivo para a sustentabilidade, tanto quanto foi para a sustentabilidade do projeto Linkin Universities in Asia and Europe in Implementing Sustainable Technology.

Ao fazer uma reflexão sobre os aspectos pedagógicos, conclui-se que, se a intenção é um resultado satisfatório, os cursos com características próximas à experiência apresentada suscitam um considerável trabalho de preparação e execução (RUMBLE, 1996; RUMBLE; INTERNATIONAL INSTITUTE FOR EDUCATIONAL, 1992; THORPE, 2001). Da mesma forma, deve ser considerado o número de pessoas implicadas (docentes, estudantes, colaboradores, técnicos e outros), sendo também necessário um calendário detalhado para a produção e execução de todas as atividades de cursos com tais características (LOCKWOOD; LATCHEM, 1998).

\section{Projeto Bättrekonceptet (O Melhor Conceito), 2002}

Uma pesquisa realizada nas 100 maiores empresas do município de Lidköping, sudoeste da Suécia, apontou que os trabalhadores necessitavam de atualização e incrementação de seus conhecimentos em técnicas de produção. A partir de então, a Universidade de Borås - HB passou a trabalhar de forma colaborativa com os Centros Universitários Regionais - CUR para implementar um programa de $\mathrm{EaD}$, visando suprir as necessidades das empresas e dos profissionais que nelas atuavam.

Para essa ocasião, a Universidade de Borås - HB teve que desenvolver uma metodologia própria, e uma conclusão para o desenvolvimento metodológico e pedagógico dos cursos foi a necessidade de dedicar mais tempo para o planejamento das atividades a serem desenvolvidas a distância do que para um projeto tradicional (DANIEL, 1996). A qualidade do curso a distância depende diretamente de que o docente tenha dedicado tempo suficiente antes que inicie as atividades, pois é por meio de um planejamento prévio que podem ser combinadas as ferramentas TIC disponíveis com 
os materiais selecionados para o desenvolvimento dos estudos (trabalho metodológico prévio).

Para evitar grandes equívocos, foi definida a adaptação de um curso piloto, oferecido na modalidade semipresencial, considerando a necessidade de que os participantes pudessem: trabalhar colaborativamente; desenvolver a capacidade de pensar criticamente, analisando problemas e soluções e desenvolvendo-se ativamente no processo de aprendizagem permanente; aprender formas de comunicação efetivas (DILLEMANS, 1998; SÖDERLUND, 2000), sem, contudo, ter a necessidade de estar fisicamente presentes na instituição sede da formação.

As aulas foram gravadas e disponibilizadas aos participantes por meio de Internet por Mediação Stream (corrente). Para os participantes que não tinham acesso à Internet ou tinham acesso limitado devido à capacidade da banda, foram oferecidas gravações em CD ou DVD, enviadas por correio.

Profissionais capacitados para dar suporte aos docentes, estudantes motivados para o emprego das ferramentas TIC e ferramentas disponíveis no SGA, constituiu-se em elemento importante, mas foram os CUR que desempenharam um papel indispensável na implementação do programa de educação semipresencial. Nos CUR, os estudantes reuniam-se uma vez por semana e, por meio da utilização de diferentes ferramentas, estabeleciam, além de relações entre si, a criação de redes entre os profissionais e as empresas nas quais atuavam.

Outro aspecto relevante é que, durante o desenvolvimento do curso, o docente estava presente por meio de videoconferência, ministrando as aulas virtualmente com o emprego do SGA e, dessa forma, os estudantes tinham acesso a orientações e informações sobre o curso, acessavam a literatura solicitada, promoviam discussões sobre seus estudos por meio de fóruns, além de outras iniciativas que formaram as possibilidades para a aprendizagem. Além das seções de videoconferência, o docente visitava os CUR, mantendo também um contato físico com os estudantes várias vezes durante o curso.

Por meio do Bättrekonceptet e do uso de um SGA, estudantes em situação laboral puderam acessar os estudos superiores sem a necessidade de abandonar o trabalho. Destaca-se ainda que esse projeto obteve grande aceitação entre os acadêmicos, sendo que, no outono europeu de 2007, foi alcançado um total de 1000 inscritos. Não foram somente as empresas e os próprios estudantes que elogiaram essa forma de educação, Bättrekonceptet também obteve prêmios nacionais e reconhecimento internacional por sua inovação pedagógica e metodológica. 


\section{Projeto Overview of Sustainable Development}

Ao finalizar o projeto Linkin Universities in Asia and Europe in Implementing Sustainable Technology, 2003 - 2006, do Programa Asia Link, financiado pela Comunidade Europeia, tomou-se, na Universidade de Borås - HB, a decisão de que todos os estudantes da instituição deveriam trabalhar conhecimentos básicos sobre desenvolvimento sustentável. Por razões de cooperação e desenvolvimento de países em vias de desenvolvimento, também se decidiu por possibilitar a estudantes de outros países o registro na formação, sem incidir gastos econômicos para a matrícula. A esses estudantes foi proposto um certificado de conclusão de Curso da Escola de Engenharia da Universidade de Borås - HB.

Durante o planejamento do curso, decidiu-se que ele seria ministrado a distância e com ajuda de um SGA, devido aos bons resultados obtidos durante a implementação de outros projetos. Uma vez identificado o grupo de estudantes e planejado o conteúdo, foi analisada a metodologia mais adequada. Três parâmetros foram identificados: existência de um grande número de interessados registrados no curso; velocidade de acesso à Internet em países em vias de desenvolvimento, que não era comparável à da Suécia; necessidade de gratuidade do acesso à literatura do curso, considerando a situação econômica de muitos alunos nesses países.

Para vencer todas as limitações existentes foi definido que: o idioma comum para todos os estudantes seria o inglês; para facilitar a gestão do curso, otimizar recursos e elevar o aproveitamento do tempo, seriam utilizadas as ferramentas que compõem um SGA, especialmente para automatizar ao máximo possível as diferentes partes do curso, como, por exemplo, a correção das avaliações; limitar-se-ia e controlar-se-ia o tamanho máximo de arquivos de informação que fossem distribuídos no decorrer do curso; toda literatura deveria ser disponibilizada aos participantes de forma simples, por meio de enlaces em Internet, pelo docente responsável pelo curso; deveriam ser disponibilizadas orientações claras sobre o funcionamento do SGA e a metodologia empregada durante a capacitação.

O Overview of Sustainable Development foi ministrado de 2007 a 2010 na Universidade de Borås - HB, com bons resultados, sendo também oferecido a outras universidades. Esse curso contribuiu para o aumento da consciência entre os estudantes da importância do desenvolvimento sustentável não somente local, mas também global. 
Programa de Adaptação da Concepção Pedagógica e da Metodologia do Ensino Superior com utilização do SGA e REA, 2012

No decorrer do ano de 2012, foi desenvolvido um programa de formação em TIC para uma equipe de docentes do Unibave, em Orleans, sul de Santa Catarina, Brasil. Oferecido gratuitamente pela Universidade de Borås - HB em função de um acordo firmado entre as duas instituições, o programa tinha como pretensão fomentar o uso das TIC no Ensino Superior, sendo, para isso, utilizado como base tecnológica um SGA de Código Aberto, especificamente, a Plataforma Moodle.

Entre as diferenças do programa estava a concepção pedagógica que o norteou, fugindo dos parâmetros tradicionais dos cursos que expõem as possibilidades e sugerem que os participantes as situem e repitam algumas atividades para aprender os mecanismos técnicos implicados no uso das ferramentas indicadas. Tendo a pesquisa e o trabalho colaborativo como aspectos balizadores da proposta pedagógica, os momentos vivenciados pelos participantes se transformaram em um processo incessante de busca e de compartilhamento das descobertas.

Considerando um de seus objetivos específicos, o programa estimulou o emprego de ferramentas que compõem um SGA, adaptando-as ao desenvolvimento do ensino virtual aplicado a estudos de caso. Para tanto, as ferramentas e a própria Plataforma Moodle foram distribuídas em memórias USB a cada um dos docentes da formação, oferecendo um acervo incomparável de possibilidades para conhecer várias ferramentas TIC e criar formas de explorá-las, de acordo com o interesse de cada participante.

Não havia, portanto, uma forma pré-determinada para cada docente adaptar sua disciplina, ofereceram-se múltiplas possibilidades para cada um projetar o seu percurso e personalizar a disciplina com as atividades e os recursos que lhe pareciam mais indicados. Além dessa abertura, a forma como o curso foi ministrado também estimulava a interação e, ao mesmo tempo que cada um aprendia a usar uma ferramenta nova, o processo de aprendizagem era compartilhado com os pares.

Ainda é cedo para avaliar os resultados do processo, que é novo tanto em termos da diversidade de ferramentas TIC - a que os docentes do curso tiveram acesso - como pela concepção pedagógica em que o programa foi desenvolvido. Contudo, apesar da necessidade da ampliação do número de laboratórios para que a Plataforma Moodle seja utilizada mais intensamente, algumas iniciativas estão comprovando que o programa de formação colhe resultados, entre os quais: a formação de uma equipe que segue estudando, adaptando, criando e compartilhando os avanços - por decisão 
dos próprios participantes do programa, essa equipe recebeu o nome de Equipe Ensino Flexível; a discussão de aspectos pedagógicos durante os encontros, além das especificidades metodológicas, contribuindo para que a inserção tecnológica fosse sustentada por teorias que estimulam o trabalho colaborativo; a elaboração de um Programa de Apoio à Aprendizagem da Física, desenvolvido pela parceria entre o Unibave e a Universidade Medellín, Colômbia, no qual os estudantes dos Cursos de Engenharia de ambas as instituições estudam de forma colaborativa na Plataforma Moodle.

\section{Considerações finais}

Não existe uma forma predefinida de utilização das TIC para adaptar o ensino às necessidades dos estudantes e do mundo do trabalho. Além disso, existe um grande risco de fracasso na implementação da $\mathrm{EaD}$ se não são consideradas as possibilidades pedagógicas do uso das ferramentas TIC e as necessidades de aprendizagem de cada contexto.

Atualmente, os docentes dispõem de novas ferramentas e métodos para levar adiante essa adaptação, ressignificação e distribuição do ensino. Um bom exemplo de adequação da educação às novas formas de aprendizagem e necessidades da sociedade é o projeto Bättrekonceptet, apresentado neste trabalho.

Em seu desenvolvimento, não foram somente os estudantes os beneficiados, mas também as empresas, com a atualização e melhora do seu pessoal técnico. Em decorrência, é a sociedade, em seu conjunto, a maior beneficiada. Nesse processo, a Aprendizagem Permanente (Life Long Learning) e a nova organização entre Graduação e Pós-Graduação podem incrementar uma aprendizagem flexível para estimular o acesso e a educação universitária, durante a vida profissional das diferentes áreas de formação.

Esse tipo de adaptação de educação e inovação pedagógica não é viável somente na Europa, mas tem uma aplicabilidade global. Precisamente, esse enfoque tem uma grande relevância para o desenvolvimento de projetos internacionais, nos quais a universidade, a sociedade e a inovação, com o emprego das TIC, podem melhorar significativamente a pertinência e a qualidade da educação.

Há mais de uma década, em muitos países, existe uma considerável pressão política para o uso das TIC na educação. Um dos exemplos é o informe da Grã-Bretanha Dearing (Comitê Nacional de Pesquisa sobre a Educação Superior), que registra na lista de recomendações que todas as instituições devem, em médio prazo, rever a evolução do papel dos funcionários, como resultado da inserção tecnológica, e garantir 
que tanto eles como os estudantes recebam formação que estimule o desenvolvimento do potencial de cada um (EDUCATION, 1997).

Em países europeus como a Suécia, especificamente, desde a segunda metade dos anos de 1990, políticas governamentais indicavam que a utilização das TIC deveria ser incrementada no Ensino Superior, para aumentar a qualidade da educação (REGERINGEN PROPOSITION, 1996). Um dos resultados dessa política foi a criação do Programa Nacional para o Emprego das TIC nas Escolas (REGERINGEN PROPOSITION, 1998).

Na prática, observa-se que existem diferentes instituições educativas fomentado que o uso das ferramentas TIC no ensino aconteça progressivamente (COLLIS; VAN DER WENDE, 2002). Contudo, realizar essa mudança e usar todo o potencial que o emprego das referidas ferramentas oferece ao Ensino Superior é mais difícil quando aos docentes é atribuída toda a carga de planejamento e execução dos cursos.

Durante as discussões com membros de projetos nos quais foram empregadas as TIC, deduziu-se que, por regra geral, os docentes consideram que não recebem o apoio suficiente para a aquisição dos conhecimentos necessários ao emprego dessas ferramentas na docência. Essa falta de conhecimento supõe um esforço individual mais profundo na hora de planejar e ministrar o curso, e o resultado é que grande parte dos docentes seguem usando uma metodologia tradicional.

Entre os fatores que dificultam a inserção tecnológica também pode estar a falta de motivação para dedicar tempo para empregar as ferramentas TIC ainda desconhecidas, entre as quais estão os Recursos Educacionais Abertos (REA). A sobrecarga de trabalho e a falta de tempo também são obstáculos que impedem ao docente desenvolver métodos mais centrados no estudante para favorecer sua aprendizagem (AL-SENAIDI; LIN; POIROT, 2009).

Para reverter esse quadro, é necessário projetar programas de formação docente que, no mínimo, estimulem a inovação pedagógica por meio de trabalhos colaborativos, o que minimiza o esforço individualizado. Portanto, para utilizar as TIC no ensino, o primeiro aspecto observado é o equilíbrio entre a utilidade e a ferramenta e sua facilidade de uso (AL-BUSAIDI; AL-SHIHI, 2010), e isso é feito com mais facilidade quando as propostas são construídas por meio do trabalho colaborativo.

Nesse processo em construção, portanto, um dos desafios das instituições educacionais é captar a atenção e o interesse dos professores para que eles desenvolvam o emprego das ferramentas TIC de forma colaborativa e percebam a relevância para a qualidade do ensino e para ações comunitárias (GARROTE JURADO, 2007). Dessa forma, poderia ser mais explorado todo potencial que as TIC oferecem para a comuni- 
cação entre estudantes, a qual pode ser decisiva tanto para aumentar as possibilidades de aprendizagem (JYOTHI et al., 2007; NAGI, et al., 2008) quanto para estimular novas propostas pedagógicas para a melhora do Ensino Superior.

É necessário também que os docentes que pretendem ministrar cursos com a ajuda das ferramentas TIC façam uma revisão da infraestrutura de que dispõe cada universidade e que desenvolvam um planejamento estratégico de emprego dessas ferramentas para cada realidade. É somente com essa adaptação à infraestrutura que se poderá chegar à criação de uma metodologia exitosa, sendo também relevante considerar todas as possibilidades para sua melhora, já que a tecnologia não para de evoluir e torna-se cada vez mais imprescindível no ensino.

Para aumentar o uso de ferramentas TIC e, em decorrência, os Recursos Educacionais Abertos (REA) no ensino, nas universidades ou faculdades, é necessário, portanto, desenvolver estratégias que incluam planos para sua aquisição, educação e ajuda. É possível que, inclusive, seja necessário nomear um responsável com conhecimentos técnicos, metodológicos e pedagógicos (CRAIG; JASSIM, 1995) para assegurar-se de que os planos se materializem, já que "Existe uma incerteza inerente entre criação e a sua realização na prática, uma vez que a prática não é o resultado do desenho, mas uma resposta a ele." (WENGER, 1998, p. 233).

E, por fim, é importante que, durante a etapa na qual o docente está desenvolvendo um projeto com TIC, mantenha-se uma constante comunicação entre os pares para que exista um processo de retroalimentação, como o que está acontecendo com os docentes que participaram do programa de formação oferecido pela Universidade de Borås - HB ao Unibave. Essa retroalimentação é necessária para que a equipe possa discutir e desenvolver suas ideias, apropriando-se de outros pontos de vista, que resultem no aumento dos conhecimentos tanto pedagógicos como metodológicos, indispensáveis para tornar os Recursos Educacionais Abertos (REA) aliados inseparáveis da pertinência e da qualidade do Ensino Superior.

\section{Referências}

AL-BUSAIDI, A.; AL-SHIHI, H. Instructors' Acceptance of Learning Management Systems: A Theoretical Framework: Communications of the IBIMA, 2010.

AL-SENAIDI, S.; LIN, L.; POIROT, J. Barriers to adopting technology for teaching and learning in Oman. Computers \& Camp. Education, n. 53, v. 3, p. 575-590. 2009. 
ALVES, L. Educação a distância: conceitos e história no Brasil e no mundo. Revista da Associação Brasileira de Educação a Distância, v. 10, p. 80-92. 2011. Disponível em: http://www.abed.org.br/revistacientifica/Revista_PDF_Doc/2011/Artigo_07.pdf. Acesso em: 14 de maio de 2012.

BLIN, F.; MUNRO, M. Why hasn't technology disrupted academics' teaching practices? Understanding resistance to change through the lens of activity theory. Comput. Educ., v. 50, n. 2, p. 475-490. 2008.

BRITAIN, S.; LIBER, O. A Framework for Pedagogical Evaluation of Virtual Learning Environments - 143 Reports: Research; 160 Tests/Questionnaires. United Kingdom - Wales: EDRS Price MF01/PC02 Plus Postage, 1999. Disponível em: <For full text: http://www.jtap .ac. uk/reports/htm/jtap-041.html>. Acesso em: 19 maio 2013.

BUSH, M. D.; MOTT, J. D. The name assigned to the document by the author. This field may also contain sub-titles, series names, and report numbers. The Transformation of Learning with Technology: Learner- Centricity, Content and Tool Malleability, and Network Effects. Educational Technology Publications, v. 49, n. 2, p. 3-20. 2009.

BUTCHER, Niel. A Basic Guide to Open Educational Resources (OER). Paris: Commonwealth of Learning e UNESCO, 2011. Disponível em: <http://www.col.org/resources/publications/Pages/detail.aspx?PID=357>. Acesso em: 17 fev. 2014.

COLLIS, B.; VAN DER WENDE, M. Models of technology and change in higher education. An international comparative survey on the current and future use of ict in education. Enschede: CHEPS - Center for Higher Education Policy Studies, 2002.

CRAIG, S.; JASSIM, H. People and project management for it. Londres: McGraw-Hill, 1995.

DANIEL, J. The mega-universities and knowledge media: technology strategies for higher education. Londres: Kogan Page, 1996.

DILLEMANS, R. New technologies for learning: contribution of ICT to innovation in education. Lovaina : Leuven University Press, 1998. 
DUTTON, W. H., CHEONG, P.; PARK, N. The social shaping of a virtual learning environment. Electronic Journal of e-Learning, v. 2, p. 1-12. 2004. Disponível em: <http:// www.ejel.org/volume-2/vol2-issue1/issue1-art3.htm>. Acesso em: 16 set. 2009.

EDUCATION., G. B. N. C.. Higher education in the learning society / national committee of inquiry into higher education; [chairman: Sir ron dearing]. Report of the national committee. p. 467, 1997.

FINDIK, D.; OZKAN, S. Identifying success factors for WBLMS use by instructors of engineering departments. In: Frontiers In Education Conference - FIE, 16. Anais... Washington: FIE, 2010.

GARROTE JURADO, R. The use of a Learning Management System to promote group interaction and socialization. In: Conferencia HSS 07. Anais... Area de "long life learning", 2007.

Barriers to a wider Implementation of LMS in Higher Education: a Swedish case study, 2006-2011. Eleed, 9. 2012.

GARROTE JURADO, R.; PETTERSSON, P. Lecturers' attitudes about the use of learning management systems in engineering education: A Swedish case study. Australasian Journal of Educational Technology, v. 23, n. 3, p. 328-350. 2007.

IRWIN, C.; BERGE, Z. Socialization in the Online Classroom. e-Journal of Instructional Science and Technology - e-JIST, v. 9, n. 1. 2006. Disponível em:

$<$ Retrieved from http://www.usq. edu.au/electpub/ejist/docs/vol9_no1/papers/full_ papers/irwin_berge.htm>. Acesso em: 19 maio 2013.

JYOTHI, S.; MCAVINIA, C.; G. KEATING, J. An Interaction Visualisation Tool for a Learning Management System. Proceedings of the 2007 conference of the center for advanced studies on Collaborative research. Ontario: Richmond Hill, 2007.

KARGIDIS, T.; STAMATIS, D.; MANITSARIS, A. Virtual Learning Institution: A Distributed Model for Networked Open Learning for the Purposes of Lifelong Learning. Journal of Information Technology Impact, v. 3, n. 1, p. 11-24. 2003. 
LADYSHEWSKY, R. K.; GARDNER, P. Peer assisted learning and blogging: A strategy to promote reflective practice during clinical fieldwork. Australasian Journal of Educational Technology, n. 24, v. 3, p. 241-257, 2008.

LEASK, M. Issues in teaching using ICT. London: Routledge Falmer, 2001.

LOCKWOOD, F.; LATCHEM, C. Staff development in open and flexible learning. Londres: Routledge, 1998.

MAIA, C.; J. MATTAR. $A B C$ da EaD: a Educação a Distância hoje. São Paulo: Pearson, 2007.

NAGI, K.; SUESAWALUK, P.; VATE U-LAN, P. Evaluating Interactivity of eLearning Resources in A Learning Management System (LMS) - A Case Study of MOODLE, An Open Source Platform. In: Conference on eLearning for Knowledge-Based Society, 23. Anais... Tailândia: Assumption Unviersity, Suvarnabhumi Campus, 2008.

NASCIMENTO, D. S.; FERREIRA, S. L. EaD no Ensino Superior público: uma política pública para democratizar o acesso ao Ensino Superior. In: Simpósio Educação e Comunicação: Infoinclusão - possibilidades de ensinar e aprender, 3. Anais... Aracaju: Universidade Tiradentes, 2012. p. 202-213. Disponível em: $<$ http://geces.com.br/simposio/anais/anais-2012/Anais-202-213.pdf $>$. Acesso em: 27 fev. 2014.

PRETI, O. (Org.). Educação a distância: sobre discursos e práticas. Brasília: Liber Livro, 2005.

REGERINGEN PROPOSITION. Regeringens proposition 1995/96:125. Åtgärder för att bredda och utveckla användningen av informationsteknik. 1996.

Regeringens skrivelse 1997/98:17: Lärandets Verktyg: Nationellt program för it i skolan, 1998.

RUMBLE, G. The costs and economics of open and distance learning. London: Kogan Page, 1996. 
SEEGER, M. A.; ÅSTRÖM, A. Sammanfattning av D-uppsats i Utbildningsdesign Distansutbildning via lärplattform - en överlevnadsstrategi? Linköping: Linköpings Universitet, 2005.

SCOTT, G. Change matters: making a difference in education and training. St. Leonards, N.S.W.: Allen \& Unwin, 1999.

SÖDERLUND, A. Lära om Lärande. Perspektiv på lärande oberoende av tid och plats. Lund: Studentlitteratur, 2000.

THORPE, M. Evaluating the use of learning technologies. In: BURGE, E.; AUGHEY, M. (Eds.). Using Learning Technologies: International Perspective on Practice. Londres: Routlede Falmer, 2001.

UBELL, R. Engineers turn to e-learning. Spectrum, IEEE, v. 37, n. 10, p. 59-63. 2000.

WILSON, G.; STACEY, E. Online interaction impacts on learning: Teaching the teachers to teach online. Australasian Journal of Educational Technology, v. 20, n. 1, p. 33-48, 2004.

XIE, K.; KE, F. The role of students' motivation in peermoderated asynchronous online discussions. British Journal of Educational Technology, n. 42, v. 6, p. 916-930. 2011.

Recebido em: 18 abril 2016

Aceito em: 25 abril 2016 
